\title{
Recurrent Pregnancy Loss in Patients with Polycystic Ovary Syndrome: A Case Control Study
}

\author{
Lulwa Ashaq ${ }^{1}$, Yousef Al Mazer' ${ }^{1}$, Nourah Al Qahtani² \\ ${ }^{1}$ Adan Hospital, Al Ahmadi, Kuwait \\ ${ }^{2}$ Feto-Maternal Medicine, Immam Abdulrahman Bin Faisal University, Dammam, Saudi Arabia \\ Email:dr_lulwa@hotmail.com, yousef-online@hotmail.com,nhqahtani@uod.edu.sa
}

How to cite this paper: Ashaq, L., Al Mazer, Y. and Al Qahtani, N. (2017) Recurrent Pregnancy Loss in Patients with Polycystic Ovary Syndrome: A Case Control Study. Open Journal of Obstetrics and Gynecology, 7, 1073-1085.

https://doi.org/10.4236/ojog.2017.711108

Received: August 27, 2017

Accepted: October 15, 2017

Published: October 18, 2017

Copyright ( $) 2017$ by authors and Scientific Research Publishing Inc. This work is licensed under the Creative Commons Attribution International License (CC BY 4.0).

http://creativecommons.org/licenses/by/4.0/

(c) (i) Open Access

\begin{abstract}
Objectives: To identify different biophysical and hormonal factors that could contribute to recurrent pregnancy loss in Saudi women who have been diagnosed with polycystic ovary syndrome. Study Design: Case-control study. Settings: Infertility clinic in Dammam Maternity and Children Hospital. Cases: PCOS patients with recurrent pregnancy loss. Controls: PCOS patients without recurrent pregnancy loss. Results: The prevalence of recurrent abortion in PCOS patients was $31 \%$. There was no significant difference between the cases and the controls in the age, 33.1 years versus 31.9 years, BMI, 31.4 $\mathrm{kg} / \mathrm{m}^{2}$ versus $31.6 \mathrm{~kg} / \mathrm{m}^{2}$ respectively. There was no significant difference in the successful pregnancies outcome in cases and controls, term deliveries $84 \%$ versus $90 \%$, preterm deliveries $10 \%$ versus $5 \%$, twin pregnancy $6 \%$ versus $5 \%$ respectively. There was no significant difference in the hormonal profile (prolactin, $\mathrm{LH}, \mathrm{FSH}, \mathrm{LH} / \mathrm{FSH}$ ratio, TSH, progesterone, testosterone) between cases and controls. Serum estradiol level was significantly lower in the cases compared to the controls, $55.5 \mathrm{pg} / \mathrm{mL}$ versus $83.9 \mathrm{pg} / \mathrm{mL}$ respectively ( $\mathrm{p}<$ 0.01). Conclusion: PCOS patients with recurrent abortions had no significant increase in the age, BMI, preterm pregnancy, multiple gestation and ectopic pregnancy. The hormonal profile (prolactin, LH, FSH, LH/FSH ratio, TSH, progesterone, testosterone) was comparable between the cases and the controls, except for serum estradiol level, which was significantly lower in PCOS patients with recurrent abortions.
\end{abstract}

\section{Keywords}

Polycystic Ovary, Recurrent Abortion 


\section{Introduction}

Recurrent pregnancy loss classically refers to the occurrence of three or more consecutive losses of clinically recognised pregnancies prior to the 20th week of gestation. Ectopic, molar, and biochemical pregnancies are not included [1]. Approximately 15 percent of pregnant women experience sporadic loss of a clinically recognized pregnancy. About 2 percent of pregnant women experience two consecutive pregnancy losses and only 0.4 to 1 percent of them have three consecutive pregnancy losses [2].

Identified risk factors for recurrent pregnancy loss include previous pregnancy loss, advanced maternal age, uterine anomalies and adhesions, cervical insufficiency, immunological diseases such as antiphospholipid syndrome and autoimmune thyroid disease, endocrine disorders such as diabetes mellitus, polycystic ovary syndrome, and hyperprolactinemia. Although in almost fifty percent of the cases the pathophysiology remains unknown, the clinical association of recurrent pregnancy loss and polycystic ovary syndrome is more than common [2].

Polycystic ovary syndrome is an endocrine disorder that typically presents with a wide spectrum of clinical features, ranging from chronic anovulation and hyperandrogenism, to polycystic ovaries morphology on ultrasound with no menstrual or biochemical abnormality.

The incidence rate between polycystic ovary syndrome and recurrent pregnancy loss remains uncertain, due to a wide variation in different studies, ranged from 10 percent in a recent study [3], and up to 80 percent in another [4].

In addition, some studies have reported that women with polycystic ovary syndrome who either hypersecrete luteinizing hormone (LH) or who are hyperandrogenemic, are at increased risk of miscarriage following spontaneous or assisted conception [5], while other researchers disagreed [6].

The objective of this study was to identify different biophysical and hormonal factors that could contribute to recurrent pregnancy loss in Saudi women diagnosed with polycystic ovary syndrome.

\section{Methods}

This was a case-control study. It was conducted in the Maternity and Children Hospital (MCH), Dammam, Eastern Province, Saudi Arabia. This is the main maternity hospital in the Eastern Province of Saudi Arabia. The total number of deliveries is around 14,000 births each year. The hospital administration and ethics and board committee approved this study.

Saudi patients diagnosed with polycystic ovary syndrome and those who were being managed for recurrent pregnancy loss and also have polycystic ovary syndrome were evaluated.

Patients attending the infertility clinic in the outpatient department of the afore-mentioned maternity were enrolled. The exclusion criteria were non-Saudi women, single women with PCOS, married nulliparous women, women with 
uterine anomalies, antiphospholipid syndrome (APS), or women with chronic diseases that could contribute to pregnancy loss.

The patients were identified by searching the medical records database for the diagnosis of PCOS, body measurements, parity and pregnancy outcome, and hormonal levels.

\subsection{Data Collection}

Data was collected from the medical records on a specifically designed Excel 2011 workbook (Microsoft Inc.).

Information was retrieved for the following variables: age, height, weight, body mass index, parity and pregnancy outcome, history of ectopic and molar pregnancy, and hormonal levels including prolactin, luteinizing hormone (LH), follicle stimulating hormone (FSH), thyroid stimulating hormone (TSH), estradiol (E2), progesterone, and testosterone.

\subsection{Data Analysis}

These variables for patients diagnosed with PCOS with recurrent pregnancy loss were compared with a control group of women with PCOS without recurrent pregnancy loss.

Statistical analysis was carried out using Excel 2011 and social science statistics website. The mean, median, mode, standard deviation, and range, were measured. For the continuous data, student t-test was used to compare the means. A p-value of less than 0.05 was considered significant.

\section{Results}

A total of 148 women were identified as PCOS. Forty-six women (31\%) had a history of recurrent abortions, and 102 women (69\%) were diagnosed as PCOS but without history of recurrent abortion.

Table 1 shows the demographic data of 46 patients with PCOS with recurrent abortions and 102 controls.

Women with PCOS with recurrent abortions were slightly older than the controls, with a mean age of 33.1 years (21 - 50) compared to 31.9 years (21 - 47), but the result was not statistically significant ( $\mathrm{p}$-value $>0.05$ ).

Height and weight were measured to calculate the body mass index (BMI). Mean height was $1.57 \mathrm{~cm}$ for the cases, and $1.56 \mathrm{~cm}$ for the controls, and the mean weight was $78.4 \mathrm{~kg}$ for the cases, and $77.9 \mathrm{~kg}$ for the controls. There was no significant difference in the BMI between the cases and the controls, 31.4 $\mathrm{kg} / \mathrm{m}^{2}(16.8-47.1)$ and $31.6 \mathrm{~kg} / \mathrm{m}^{2}(17.0-49.1)$ respectively (p-value $\left.>0.05\right)$.

Table 2(a) compares the parity and pregnancy outcome in both cases and controls. A total number of 211 pregnancies were identified in the cases, and 198 pregnancies were identified in the controls.

A total 26/211 term deliveries (12\%) were identified in the cases, compared to $102 / 198$ term deliveries $(51 \%)$ in the control ( $\mathrm{p}$-value $<0.05$ ). 
Table 1. Demographic data mean (Range).

\begin{tabular}{|c|c|c|c|c|c|}
\hline & CASES & & CONTROL & & p-value \\
\hline Number (n) & 46 & & 102 & & - \\
\hline Age (years) & 33.1 & $(21-50)$ & 31.9 & $(21-47)$ & NS \\
\hline Height (m) & 1.57 & & 1.56 & & - \\
\hline Weight (kg) & 78.4 & & 77.9 & & - \\
\hline BMI $\left(\mathrm{kg} / \mathrm{m}^{2}\right)$ & 31.4 & $(16.8-47.1)$ & 31.6 & $(17.0-49.1)$ & NS \\
\hline
\end{tabular}

Table 2. (a) Parity and outcome number (Percentage); (b) Successful pregnancy number (Percentage).

(a)

\begin{tabular}{cccccc}
\hline & CASES (n) & $\%$ & CONTROL (n) & $\%$ & p-value \\
\hline Term & 26 & -12.3 & 102 & -51.5 & $<0.05$ \\
Preterm & 3 & -1.4 & 6 & -3 & NS \\
Twin & 2 & -0.9 & 6 & -3 & NS \\
Abortion & 174 & -82.4 & 77 & -38.8 & $<0.001$ \\
Ectopic & 3 & -1.4 & 7 & -3.5 & NS \\
Molar & 3 & -1.4 & 0 & 0 & - \\
Total & 211 & $100 \%$ & 198 & $100 \%$ & 409 \\
\hline
\end{tabular}

(b)

\begin{tabular}{ccccc}
\hline & CASES (n) & $\%$ & CONTROL (n) & $\%$ \\
\hline Term & 26 & -84 & 102 & -90 \\
Preterm & 3 & -10 & 6 & -5 \\
Twin & 2 & -6 & 6 & -5 \\
Total & 31 & $100 \%$ & 114 & $100 \%$ \\
\hline
\end{tabular}

There was no significant increase in preterm deliveries in either cases or controls, $1.4 \%(3 / 211)$ and $3 \%(6 / 198)$ respectively, and there was no significant difference either ( $p$-value $>0.05)$.

There was no significant increase in the rate of twin pregnancy in cases and controls, $0.9 \%(2 / 211)$ and $3 \%(6 / 198)$ respectively. There was no significant difference either ( $\mathrm{p}$-value $>0.05)$.

A total of $174 / 211$ abortions (82\%) were identified in the cases, compared to $77 / 198$ abortions $(38 \%)$ in the controls, and it was very highly significant (p-value $<0.001$ ).

The overall prevalence of ectopic pregnancy among cases and controls was $2.4 \%(10 / 409)$. The rate of ectopic pregnancies in the cases and the controls was $1.4 \%(3 / 211)$ and $3.5 \%(7 / 198)$ per total parity respectively. There was no significant difference ( $\mathrm{p}$-value $>0.05$ ). Three episodes of molar pregnancy were iden- 
tified in a single patient in the cases group only.

Table 2(b) is a subdivision of Table 2(a) to assess the outcome of successful pregnancy. $84 \%(26 / 31)$ of the cases and $90 \%(102 / 114)$ of the controls had term pregnancies. The incidence of preterm delivery was double in the cases compared to control, $10 \%(3 / 31)$ versus $5 \%(6 / 114)$ respectively. The rate of twin pregnancy was similar between the cases and the controls where it was $6 \%(2 / 31)$ versus $5 \%(6 / 114)$ in the cases compared to the controls.

Table 3 compares the mean hormonal level between the cases and the controls.

There was no significant difference in the mean prolactin level between the cases and the controls, $16.7(4.68$ - 75.4) and 17.7 (0.76 - 297) respectively ( $\mathrm{p}$-value $>0.05$ ).

Luteinizing hormone (LH) level was 7.18 (1.06 - 75.4) in the cases compared to $6.93(1.29-26.2)$ in the controls. The result was not statistically significant (p-value $>0.05)$.

Follicle-stimulating hormone (FSH) level was $6.54(0.75$ - 16.2) in the cases, compared to $6.24(2.7-23.5)$ in the controls. The result was no statistically significant ( $\mathrm{p}$-value $>0.05$ ). In addition, there was no significant difference in the LH/FSH ratio between the cases and the controls, 1.29 and 1.17 respectively (p-value $>0.05)$.

There was no significant difference in the mean thyroid-stimulating hormone (TSH) level between the cases 2.57 (0.86 - 6.25), and the controls 2.48 (0.4614.4) (p-value >0.05).

The mean estradiol (E2) level was slightly lower in the cases compared to the controls, 55.5 (13.1 - 224) and 83.9 (0.83 - 482) respectively. The difference was statistically significant ( $\mathrm{p}$-value $<0.01$ ). Pearson correlation coefficient for estradiol (R-value) was -0.188 .

Mean progesterone level was 11.04 (0.1 - 25.3) in the cases, compared to 9.50 $(0.1$ - 35.8) in the controls. The difference was not statistically significant (p-value $>0.05)$.

Table 3. Hormonal levels mean (Range).

\begin{tabular}{cccccc}
\hline & $\begin{array}{c}\text { CASES } \\
\text { (mean) }\end{array}$ & & $\begin{array}{c}\text { CONTROL } \\
(\text { mean })\end{array}$ & p-value \\
\hline Prolactin (ng/mL) & 16.7 & $(4.68-75.4)$ & 17.7 & $(0.76-297)$ & NS \\
LH (IU/L) & 7.18 & $(1.06-28.2)$ & 6.93 & $(1.29-26.2)$ & NS \\
FSH (IU/L) & 6.54 & $(0.75-16.2)$ & 6.24 & $(2.7-23.5)$ & NS \\
LH/FSH Ratio & 1.29 & $(0.145-6.86)$ & 1.17 & $(0.247-4.93)$ & NS \\
TSH ( $\mu$ IU/L) & 2.57 & $(0.86-6.25)$ & 2.48 & $(0.46-14.4)$ & NS \\
Estradiol (pg/mL) & 55.5 & $(13.1-224)$ & 83.9 & $(0.83-482)$ & $<0.01$ \\
Progesterone (ng/mL) & 11.04 & $(0.1-25.3)$ & 9.5 & $(0.1-35.8)$ & NS \\
Testosterone (ng/dL) & 6.22 & $(0.23-58.9)$ & 8.5 & $(0.08-126.4)$ & NS \\
\hline
\end{tabular}


The mean testosterone level was slightly lower in the cases compared to the controls, $6.22(023$ - 58.9) and 8.50 (0.08 - 126.4), but the difference was not statistically significant ( $\mathrm{p}$-value $>0.05)$.

\section{Discussion}

The association between PCOS and recurrent pregnancy loss has been studied in many literatures since a couple of decades. Most researchers studied PCOS patients and compared them to normal controls. In our study, we divided the women who have been diagnosed with PCOS into two groups, PCOS with recurrent abortions and PCOS without recurrent abortions. The prevalence of recurrent abortions varied widely in different literatures. In an old historic study by Sagle et al., they reported that $80 \%$ of PCOS women had recurrent pregnancy loss [4], whereas more recent studies reported a prevalence of 30 to 40 percent [6]. Homburg et al. studied the prevalence of recurrent pregnancy loss among PCOS and non-PCOS patients who underwent ovulation induction. He reported a prevalence of $33 \%$ among PCOS patients, compared to $10 \%$ in non-PCOS women [6]. Rai et al. and Banu et al. also reported a prevalence of recurrent abortion of $40 \%$ among women with PCOS compared to normal controls [7] [8].

In our study, we found the prevalence of recurrent pregnancy loss among women diagnosed with PCOS to be $31 \%$, which is close to most reports.

Maternal age is an important factor for the prediction of possible pregnancy loss. Unfortunately, we could not find many reports comparing maternal age in two groups of PCOS with and without recurrent pregnancy loss. Most reports studied the prevalence of pregnancy loss in association with advanced maternal age. Literatures reported that the risk increases significantly after 35 years of age. In a large Scandinavian study by Andersen et al., they found that women older than 35 years of age had 25 percent risk of spontaneous abortion [9]. McClure et al. studied the association of serum estradiol level, maternal age, and pregnancy outcome in PCOS patients. They found that increased maternal age was associated with lower serum estradiol level, which in turn, was significantly associated with recurrent miscarriage [10]. In our study, the mean maternal age was below 35 years for both cases and controls. In addition, we did not find any significant difference in the mean age between cases and controls, 33.1 years versus 31.9 years respectively.

Obesity has been conclusively associated with an increased prevalence of miscarriage, and obesity is obviously more common in PCOS patients than in normal controls [6]. Wang et al. found that a higher rate of spontaneous miscarriage in PCOS patients was due to higher prevalence of obesity [11]. In addition, Boots et al. found that obese women are more prone to have euploid miscarriage compared to non-obese controls [12]. Obesity is a common finding in Saudi women due to dietary habits and personal lifestyle. When Fakhoury et al. compared PCOS patients to normal controls in Saudi women; they found both groups to have BMI above $25 \mathrm{~kg} / \mathrm{m}^{2}$ [13]. Similar to Fakhoury et al., we found that both groups of PCOS cases and controls having a BMI above $30 \mathrm{~kg} / \mathrm{m}^{2}$. On the other 
hand, there was no significant difference in the BMI between both groups where it was $31.4 \mathrm{~kg} / \mathrm{m}^{2}$ among cases, and $31.6 \mathrm{~kg} / \mathrm{m}^{2}$ among controls.

Many researchers have studied adverse obstetric outcome in PCOS patients, apart from pregnancy loss. In a recent meta-analysis by Qin et al., they found that PCOS patients had higher incidence of pregnancy induced hypertension, gestational diabetes, preterm delivery, and Caesarean section [14]. In addition, Naver et al. reported a doubled incidence of preterm delivery and pre-eclampsia in hyper-androgenic women with PCOS compared to normo-androgenic PCOS [15]. In our study, most of the cases and controls who delivered, had a term pregnancy, 84 percent versus 90 percent, respectively. On the other hand, similar to what was observed by Naver et al., the rate of preterm deliveries among the cases was twice as much as the controls, 10 percent versus 5 percent, respectively. Multiple gestation is a possible complication of induction of ovulation as a treatment of the anovulatory cycles in PCOS patients. Nahuis et al. reported an incidence of twin pregnancy in PCOS patients in a randomised trial as 5 percent after being treated by laparoscopic electrocautery, and 8 percent after receiving recombinant-FSH [16]. In addition, Zhao et al. studied the pregnancy outcome after in vitro maturation of immature oocytes, and found a significant increase in multiple gestation up to 30 percent, low birth weight, and preterm deliveries [17]. In our study, we did not find a significant increase in the rate of twin pregnancy in either cases or controls. Similar to what was observed by Nahuis et al., the rate of twin pregnancy, out of the successful deliveries, was comparable between cases and controls, 6 versus 5 percent respectively. There are few reports studied the incidence of ectopic pregnancy in PCOS patients. Wang et al. studied the incidence of ectopic pregnancy after in vitro fertilization in PCOS patients, and reported a threefold increase in the incidence of ectopic pregnancy in PCOS patients compared to normal controls specially after fresh embryo transfer [18]. In our study, the overall prevalence of ectopic pregnancy among cases and controls was 2.4 percent, which is slightly higher than general population, twenty per thousand [19]. In addition, the cases had slightly higher incidence of ectopic pregnancy than the controls, 8 percent versus 6 percent. There were 3 incidents of molar pregnancy in a single patient in the cases group.

As previously known, polycystic ovary syndrome is an endocrine disorder that typically present with a wide spectrum of clinical features. Many literatures have studied the association between different endocrinopathies and recurrent miscarriage that present in PCOS and non-PCOS patient. Most researchers studied the effect of hyperprolactinemia, subclinical hypothyroidism, abnormal luteinizing and follicle stimulating hormones, abnormal progesterone level, and hyperandrogenism. In our study we measured these hormones, and compared them between the cases and the controls.

Many researchers have noticed the association between hyperprolactinemia and early pregnancy loss. In a randomized controlled trial by Hirahara et al., they evaluated the effect of isolated hyperprolactinemia in the pathogenesis of recurrent spontaneous abortion. They found that restoration of normal prolactin 
level with bromocriptine was associated with a significant improvement in the pregnancy outcome, and concluded that appropriate circulating levels of prolactin may play an important role in maintaining early pregnancy, especially in cases of hyperprolactinemic recurrent miscarriage [20]. On the other hand, Li et al. found that even low basal serum prolactin level was associated with early pregnancy loss [21]. In our study, the mean serum prolactin level was comparable between the cases and the control, $16.7 \mathrm{ng} / \mathrm{mL}$ versus $17.7 \mathrm{ng} / \mathrm{mL}$ respectively, and it was within normal limits.

Luteinizing hormone (LH) and follicle-stimulating hormone (FSH) plays a critical role in the hormonal secretion, ovulation, and endometrial integrity. There is a controversy between literatures regarding the association between abnormal gonadotropin levels and pregnancy outcome.

It was previously thought that elevated level of luteinizing hormone in PCOS patient was associated with recurrent miscarriage [5]. However, many researchers found no significant association between serum LH concentration and recurrent miscarriage [10] [22] [23]. In a randomized control trial by Clifford et al., they suppressed pituitary LH release and observed its effect on patients with recurrent pregnancy loss. They concluded that pre-pregnancy suppression of high luteinizing hormoneconcentrations in ovulatory women with recurrent miscarriage and hypersecretion of luteinizing hormone does not improve the outcome of pregnancy [24]. On the other hand, some literatures found a significant association between elevated day 3 FSH level in women with recurrent unexplained miscarriage [25] [26]. In addition, Fakhoury et al. reported an elevated level of LH/FSH ratio in PCOS patients compared to normal controls [13]. In our study, we found no significant difference between the cases and the controls regarding mean LH, FSH levels, and LH/FSH ratio, $7.18 \mathrm{IU} / \mathrm{L}$ versus $6.93 \mathrm{IU} / \mathrm{L}$, $6.54 \mathrm{IU} / \mathrm{L}$ versus $6.24 \mathrm{IU} / \mathrm{L}, 1.29$ versus 1.17 respectively.

Thyroid hormones are essential for the normal fetal metabolism and growth. Hypothyroidism was thought to be associated with recurrent early pregnancy loss. In a study by Jacob involved one thousand women, he reported that even mild thyroid dysfunction could greatly increase the risk of serious problems. Women with mild thyroid dysfunction had double the risk of miscarriage, premature labor, and low birth weight as compared to normal controls [27]. In our study, there was no significant difference in the thyroid-stimulating hormone (TSH) level between cases and controls, and there was no suspicion of subclinical hypothyroidism. There was a controversy in literatures regarding the association of serum estradiol level and current pregnancy loss. Gurbuz et al. and Trout \&Seifer reported an elevation of serum estradiol level in women with recurrent pregnancy loss [25] [26]. On the other hand, McClure et al. found that PCOS patients with recurrent pregnancy loss had lower serum estradiol level [10]. Evrim et al. when studied the association of LH level and thyroid volume in PCOS patients, also reported a low normal serum estradiol level in PCOS patients in comparison to normal controls [28]. Similar to McClure et al. and Evrim et al., in our study we found that PCOS patients with recurrent abortion 
having lower normal serum estradiol level compared to PCOS without recurrent abortion, and it was statistically significant. In addition, serum estradiol level was negatively correlated with the incidence of pregnancy loss.

The association between serum progesterone and recurrent pregnancy loss was studied in few literatures, last was by Yan et al. who found mid-luteal serum progesterone measurement does not predict the outcome of a subsequent pregnancy in women with unexplained recurrent miscarriage [29].

Some literatures found positive correlation between hyperandrogenemia and recurrent abortion. Banu et al., Tulppala et al., and Okon et al., have found that elevated serum testosterone and other androgen level was associated with recurrent pregnancy loss in PCOS and non-PCOS patients [8] [23] [30]. On the other hand, Nardo et al. and Lathi et al. found no relation between serum testosterone level and recurrent miscarriage in PCOS and non-PCOS patients [22] [31]. In our study, serum testosterone level was higher in the control group compared to the cases, and the difference was not statistically significant.

Some researchers studied the effect of serum estradiol and pregnancy outcome in non-PCOS cases such as normal women, in-vitro fertilisation cases (IVF), and women undergoing intra-cytoplasmic sperm injection (ICSI). In three different studies by Check et al., Takeuchi et al., and Aksoy et al., serum estradiol level was measured in women with history of missed abortion or an embryonic pregnancy and compared to normal controls. All of these studies found that women with history of missed abortion had lower serum estradiol level compared to normal controls [32] [33] [34].

Other researchers found that women with recurrent abortion had significantly lower serum progesterone level compared to normal controls [33] [34]. In our study, we found no significant difference in the serum progesterone level between cases and controls.

One of the most important manifestations of PCOS is hyperandrogenism, and many literatures studied the relationship of increased androgens in PCOS patients and pregnancy outcome. Fujimoto et al. and Joo et al. focused on the effect of serum estradiol level and pregnancy outcome in IVF cases. Fujimoto et al. found that higher serum estradiol level was associated with better pregnancy outcome, while Joo et al. found a concentration-dependent effect between serum estradiol and pregnancy outcome [35] [36]. There are two studies by Rehman et al. studied the relationship between obesity, serum estradiol level, and pregnancy outcome in ICSI cases. They found that lower serum estradiol level had adverse effect on pregnancy outcome, and high peak estradiol with maintenance of optimal levels in mid-luteal phase is required for implantation of fertilized ovum and accomplishment of clinical pregnancy [37] [38]. Carranza-Lira et al. and Salazar and Calzada measured serum estradiol and progesterone levels in women with recurrent miscarriages in addition to endometrial receptors for estrogen and progesterone. They found that both estrogen and progesterone receptors were at their lowest level in the cytosol and nuclear compartment in women with recurrent miscarriage compared to normal controls [39] [40]. 
The limitations in our study was the sample size, where we had 46 cases and 102 controls over the period studied. Also, dehydroepiandrosterone (DHEA) was not routinely measured in either cases or controls.

\section{Conclusion}

PCOS patients with recurrent abortions had no significant increase in the age, BMI, preterm pregnancy, multiple gestation and ectopic pregnancy. The hormonal profile (prolactin, LH, FSH, LH/FSH ratio, TSH, progesterone, testosterone) was comparable between the cases and the controls, except for serum estradiol level, which was significantly lower in PCOS patients with recurrent abortions.

\section{References}

[1] Tulandi, T. and Al-Fozan, H.M. (2013) Definition and Etiology of Recurrent Pregnancy Lost. http://www.uptodate.com/

[2] Salat-Baroux, J. (1988) Recurrent Spontaneous Abortions. Reproduction, Nutrition, Development, 28, 1555. https://doi.org/10.1051/rnd:19881002

[3] Cocksedge, K.A., Saravelos, S.H., Metwally, M., et al. (2009) How Common Is Polycystic Ovary Syndrome in Recurrent Miscarriage. Reproductive Biomedicine Online, 19, 572-576. https://doi.org/10.1016/j.rbmo.2009.06.003

[4] Sagle, M., Bishop, K., Ridley, N., et al. (1988) Recurrent Early Miscarriage and Polycystic Ovaries. British Medical Journal, 297, 1027-1028. https://doi.org/10.1136/bmj.297.6655.1027

[5] Stanger, J.D. and Yovich, J.L. (1985) Reduced in- Vitro Fertilization of Human Oocyte from Patients with Raised Basal Luteinizing Hormone Levels during the Follicular Phase. British Journal of Obstetrics and Gynecology, 92, 385-393. https://doi.org/10.1111/j.1471-0528.1985.tb01113.x

[6] Homburg, R. (2006) Pregnancy Complications in PCOS. Best Practice \& Research Clinical Endocrinology \& Metabolism, 20, 281-292. https://doi.org/10.1016/j.beem.2006.03.009

[7] Rai, R., Backos, M., Rushworth, F. and Regan, L. (2000) Polycystic Ovaries and Recurrent Miscarriage-A Reappraisal. Human Reproduction, 15, 612-615. https://doi.org/10.1093/humrep/15.3.612

[8] Banu, J., Fatima, P., Sultana, P., Chowdury, M.A., Begum, N., Anwary, S.A., Ishrat, S., Deeba, F. and Begum, S.A. (2014) Association of Infertile Patients Having Polycystic Ovarian Syndrome with Recurrent Miscarriage. Mymensingh Medical Journal, 23, 770-773.

[9] Nybo Andersen, A.M., Wohlfahrt, J., Christens, P., Olsen, J. and Melbye, M. (2000) Maternalage and Fetal Loss: Population Based Register Link Age Study. British Medical Journal, 320, 1708.

[10] McClure, N., McDonald, J., Kovacs, G.T., Healy, D.L., McCloud, P.L., McQuinn, B. and Burger, H.G. (1993) Age and Follicular Phase Estradiol Are Better Predictors of Pregnancy Outcome Than Luteinizing Hormone in Menotropin Ovulation Induction for Anovulatory Polycystic Ovarian Syndrome. Fertility and Sterility, 59, 729-733. https://doi.org/10.1016/S0015-0282(16)55850-6

[11] Wang, J.X., Davies, M.J. and Norman, R.J. (2001) Polycystic Ovarian Syndrome and the Risk of Spontaneous Abortion Following Assisted Reproductive Technology 
Treatment. Human Reproduction, 16, 2606-2609. https://doi.org/10.1093/humrep/16.12.2606

[12] Boots, C.E., Bernardi, L.A. and Stephenson, M.D. (2014) Frequency of Euploid Miscarriage Is Increased in Obese Women with Recurrent Early Pregnancy Loss. Fertility and Sterility, 102, 455-459.

[13] Fakhoury, H., Tamim, H., Ferwana, M., Siddiqui, I., Adham, M. and Tamimi, W. (2012) Age and BMI Adjusted Comparison of Reproductive Hormones in PCOS. Journal of Family Medicine and Primary Care, 1, 132. https://doi.org/10.4103/2249-4863.104984

[14] Qin, J.Z., Pang, L.H., Li, M.J., Fan, X.J., Huang, R.D. and Chen, H.Y. (2013) Obstetric Complications in Women with Polycycstic Ovary Syndrome: A Systematic Review and Meta-Analysis. Reproductive Biology and Endocrinology, 11, 56. https://doi.org/10.1186/1477-7827-11-56

[15] Naver, K.V., Grinsted, J., Larsen, S.O., Hedley, P.L., Jorgensen, F.S., Christiansen, M. and Nilas, L. (2014) Increase Risk of Preterm Delivery and Pre-Eclampsia in Women with Polycystic Ovary Syndrome and Hyperandrogenemia. British Journal of Obstetrics and Gynaecology, 121, 575-581. https://doi.org/10.1111/1471-0528.12558

[16] Nahuis, M.J., Kose, N., Bayram, N., van Dessel, H.J., Braat, D.D., Hamilton, C.J., Hompes, P.G., Bossuyt, P.M., Mol, B.W., van der Veen, F. and van Wely, M. (2011) Long-Term Outcomes in Women with Polycystic Ovary Syndrome Initially Randomized to Receive Laparoscopic Electrocautery of the Ovaries or Ovulation Induction with Gonadotrophins. Human Reproduction, 26, 1899-1904. https://doi.org/10.1093/humrep/der141

[17] Zhao, J.Z., Chen, X., Wang, P.Y., Zhou, W., Lin, J.J., Zhang, W., Huang, X.F., Lin, W.Q., Yang, H.Y. and Chen, Y. (2009) Outcome of Pregnancy in Women with Polycystic Ovary Syndrome Treated by in Vitro Maturation of Immature Oocytes. Chinese Journal of Obstetrics and Gynaecology, 44, 409-412.

[18] Wang, J., Wei, Y., Diao, F., Cui, Y., Mao, Y., Wang, W. and Liu, J. (2013) The Association between Polycystic Ovary Syndrome and Ectopic Pregnancy after in Vitro Fertilization and Embryo Transfer. American Journal of Obstetrics and Gynecology, 209, 139.

[19] Tulandi, T. (2013) Ectopic Pregnancy: Incidence, Risk Factors, and Pathology. http://www.uptodate.com

[20] Hirahara, F., Andoh, N., Sawai, K., Hirabuki, T., Uemura, T. and Minaguchi, H. (1998) Hyperprolactinemic Recurrent Miscarriage and Results of Randomized Bromocriptine Treatment Trials. Fertility and Sterility, 70, 246-252.

[21] Li, W., Ma, N., Laird, S.M., Ledger, W.L. and Li, T.C. (2013) The Relationship between Serum Prolactin Concentration and Pregnancy Outcome in Women with Unexplained Recurrent Miscarriage. Journal of Obstetrics and Gynaecology, 33, 285-288. https://doi.org/10.3109/01443615.2012.759916

[22] Nardo, L.G., Rai, R., Backos, M., El-Gaddal, S. and Regan, L. (2002) High Serum Luteinizing Hormone and Testosterone Concentrations Do Not Predict Pregnancy Outcome in Women with Recurrent Miscarriage. Fertility and Sterility, 77, 348-352.

[23] Tulppala, M., Stenman, U.H., Cacciatore, B. and Ylikorkala, O. (1993) Polycystic Ovaries and Levels of Gonadotrophins and Androgens in Recurrent Miscarriage: Prospective Study in 50 Women. British Journal of Obstetrics and Gynaecology, 100, 348-352. https://doi.org/10.1111/j.1471-0528.1993.tb12978.x

[24] Clifford, K., Rai, R., Watson, H., Franks, S. and Regan, L. (1996) Does Suppressing 
Luteinizing Hormone Secretion Reduce the Miscarriage Rate? Results of a Randomized Controlled Trial. British Medical Journal, 312, 1508-1511. https://doi.org/10.1136/bmj.312.7045.1508

[25] Gürbüz, B., Yalti, S., Ozden, S. and Ficicioglu, C. (2004) High Basal Estradiol Level and FSH/LH Ratio in Unexplained Recurrent Pregnancy Loss. Archives of Gynecology and Obstetrics, 270, 37-39. https://doi.org/10.1007/s00404-003-0490-0

[26] Trout, S.W. and Seifer, D.B. (2000) Do Women with Unexplained Recurrent Pregnancy Loss Have Higher Day 3 Serum FSH and Estradiol Values? Fertility and Sterility, 74, 335-337.

[27] Jubbin, J.J. (2013) Subclinical Hypothyroidism in the First Trimester of Pregnancy in North India. Indian Journal of Endocrinology and Metabolism, 17, 160-161.

[28] Calir, E., Sahin, M., Topaloglu, O., Colak, N.B., Karbek, B., Gungunes, A., Arslan, M.S., Unsal, I.O., Tutal, E., Ucan, B. and Delibasi, T. (2012) The Relationship between LH and Thyroid Volume in Patients with PCOS. Journal of Ovarian Research, 5, 43. https://doi.org/10.1186/1757-2215-5-43

[29] Yan, J., Liu, F., Yuan, X., Saravelos, S.H., Cocksedge, K. and Li, T.C. (2013) Midluteal Serum Progesterone Concentration Does Not Predict the Outcome of Pregnancy in Women with Unexplained Recurrent Miscarriage. Reproductive Biomedicine Online, 26, 138-141.

[30] Okon, M.A., Laird, S.M., Tuckerman, E.M. and Li, T.C. (1998) Serum Androgen Levels in Women Who Have Recurrent Miscarriages and Their Correlation with Markers of Endometrial Function. Fertility and Sterility, 69, 682-690.

[31] Lathi, R.B., Dahan, M.H., Reynolds-May, M.F., Milki, A.A., Behr, B. and Westphal, L.M. (2014) The Role of Serum Testosterone in Early Pregnancy Outcome: A Comparison in Women with and without Polycystic Ovary Syndrome. Journal of Obstetrics and Gynaecology Canada, 36, 811-816.

[32] Check, J.H., Lurie, D., Davies, E. and Vetter, B. (1992) Comparison of the First Trimester Serum Estradiol Levels in Aborters versus Nonaborters during Maintenance of Normal Progesterone Levels. Gynecology and Obstetric Investigation, 34, 206-210. https://doi.org/10.1159/000292762

[33] Takeuchi, T., Nishii, O., Okamura, T., Yaginuma, T. and Kawana, T. (1993) Free Testosterone and Abortion in Early Pregnancy. International Journal of Gynaecology and Obstetrics, 43, 151-156.

[34] Aksoy, S., Celikkanat, H., Senöz, S. and Gökmen, O. (1996) The Prognostic Value of Serum Estradiol, Progesterone, Testosterone, and Free Testosterone Levels in Detecting Early Abortions. European Journal of Obstetrics and Gynecology, and Reproductive Biology, 67, 5-8.

[35] Fujimoto, A., Osuga, Y., Fujiwara, T., Yano, T., Tsutsumi, O., Momoeda, M., Kugu, K., Koga, K., Morita, Y., Wada, O. and Taketani, Y. (2002) Human Chorionic Gonadotropin Combined with Progesterone for Luteal Support Improves Pregnancy Rate in Patients with Low Late-Midluteal Estradiol Levels in IVF Cycles. Journal of Assisted Reproduction and Genetics, 19, 550-554. https://doi.org/10.1023/A:1021207014429

[36] Joo, B.S., Park, S.H., An, B.M., Kim, K.S., Moon, S.E. and Moon, H.S. (2010) Serum Estradiol Levels during Controlled Ovarian Hyperstimulation Influence the Pregnancy Outcome of in Vitro Fertilization in a Concentration-Dependent Manner. Fertility and Sterility, 93, 442-446.

[37] Rehman, R., Hussain, Z. and Faraz, N. (2012) Effect of Estradiol Levels on Pregnancy Outcome in Obese Women. Journal of Ayub Medical Collage, 24, 3-5. 
[38] Rehman, R., Hussain, Z., Zahir, H. and Khan, R. (2014) Impact of Peak/Mid Luteal Estradiol on Pregnancy Outcome after Intracytoplasmic Sperm Injection. The Journal of the Pakistan Medical Association, 64, 780-784.

[39] Carranza-Lira, S., Blanquet, J., Tserotas, K. and Calzada, L. (2000) Endometrial Progesterone and Estradiol Receptors in Patients with Recurrent Early Pregnancy Loss of Unknown Etiology. Medical Science Monitor, 6, 759-762.

[40] Salazar, E.L. and Calzada, L. (2007) The Role of Progesterone in Endometrial Estradiol- and Progesterone-Receptor Synthesis in Women with Menstrual Disorders and Habitual Abortion. Gynecological Endocrinology, 23, 222-225.

https://doi.org/10.1080/09513590701254030 